\title{
Effects of kinesiotape on pain, range of motion, and functional status in patients with osteoarthritis: a randomized controlled trial
}

\author{
Maryam Abolhasani, ${ }^{1,2}$, Farzin Halabchi' ${ }^{1,2}$, Roshanak Honarpishe ${ }^{3}$, Joshua A. Cleland ${ }^{4}$, Azadeh Hakakzadeh ${ }^{1,2, *}$ \\ ${ }^{1}$ Sports Medicine Research Center, Neuroscience Institute, Tehran University of Medical Sciences, Tehran, Iran \\ 2Department of Sports and Exercise Medicine, School of Medicine, Tehran University of Medical Sciences, Tehran, Iran \\ ${ }^{3}$ Department of Physical Therapy, Franklin Pierce University, Manchester, New Hampshire, USA \\ ${ }^{4}$ Department of Sport, Health \& Exercise Science, University of Hull, Kingston-upon-Hull, UK
}

Journal of Exercise Rehabilitation 2019;15(4):603-609

https://doi.org/10.12965/jer.1938290.145

In this article, the 3rd and 4th authors' affiliations were misprinted unintentionally. The affiliations should be corrected as follows:

\section{Corrected the 3rd and 4th authors' affiliations}

${ }^{1}$ Sports Medicine Research Center, Neuroscience Institute, Tehran University of Medical Sciences, Tehran, Iran

${ }^{2}$ Department of Sports and Exercise Medicine, School of Medicine, Tehran University of Medical Sciences, Tehran, Iran

${ }^{3}$ Department of Physiotherapy, School of Rehabilitation, Tehran University of Medical Sciences, Tehran, Iran

${ }^{4}$ Department of Physical Therapy, Franklin Pierce University, Manchester, NH, USA 